\title{
DIRECTED ENERGY TRANSFER DUE TO ORIENTATIONAL BROADENING OF ENERGY LEVELS IN PHOTOSYNTHETIC PIGMENT SOLUTIONS
}

\author{
A.N. RUBINOV, E.I. ZEN'KEVICH, N.A. NEMKOVICH \\ and V.I. TOMIN \\ Institute of Physics, Byelorussian Academy of Sciences, Minsk, 220602, USSR
}

Received 1981 March 1

\begin{abstract}
The directed non-radiative energy transfer through monomeric molecules of chlorophyll " $a$ " and pheophytin " $a$ " at high concentrations $\left(c \sim 10^{-2} \mathrm{M}\right)$ in a rigid matrix of polyvinylbutyral has been found by using the nanosecond laser spectrofluorimeter. The phenomenon is caused by orientational broadening of pigment molecular spectra owing to its interaction with a solvent. The observed temporal shift of the luminescence spectrum to the red region in a nanosecond time scale as well as the red shift of the time integrated spectrum at a high concentration of pigment molecules and the monotonic growth of the luminescence lifetime with a shift to the red region of the spectrum served as indications of the directed energy transfer in the sample. The non-radiative energy transfer from monomeric molecules towards aggregates is also directly demonstrated by the deformation of instantaneous luminescence spectra in the long-wavelength range $(\lambda>700 \mathrm{~nm})$. The role and the possibility of the directed energy transfer between molecules with orientationally broadened spectra in the biological systems are discussed.
\end{abstract}

\section{Introduction}

The universal (Van der Waals) intermolecular interactions (UIMI) of complex molecules with an environment are known to be responsible for a shift of the electronic states of a molecule and consequently for a molecular absorption and luminescence spectra shift in solution [1]. The magnitude of this shift for dipole molecules in polar solutions depends on the environment orientational configuration which is responsible for the local electric field interacting with a molecule. The energy of a pure electronic transition of a molecule in this case is given by

$h \nu_{\mathrm{el}}=h \nu_{0}-\Delta \boldsymbol{p} \cdot \boldsymbol{\sigma} \cos \alpha$,

where $h \nu_{0}$ is the energy gap between the ground state and the excited state of a free molecule, $\Delta p$ the change in the dipole moment of the molecule after transition to the excited state $(\Delta p>0)$, and $\alpha$ the angle between the electric 
field direction and the dipole moment. Statistical fluctuations of the environment orientational configuration should be accompanied by a change in $\delta$ and, consequently, in the frequency of the pure electronic transition $\nu_{\mathrm{el}}$. As a result, all electronic vibrational states are orientationally broadened and the absorption and emission spectra of the complex molecules appear to be a superposition of the "elementary" spectra corresponding to the transitions between the separate orientational sub-levels [2]. In a rigid matrix where the orientational relaxation time of the solvates, $\tau_{\mathrm{r}}$, is greater than the lifetime of the luminescent molecule, $\tau$, the orientational spectral broadening of inhomogeneous character is observed and is interpreted as showing the dependence of fluorescence band position on the excitation frequency when exciting in the anti-Stokes spectral range (the so-called bathochromic luminescence effect [3]). The inhomogeneous orientational broadening of the energy levels in the rigid dye solutions at high concentrations considerably influences the character of the non-radiative energy migration. Because of the difference in the overlapping integrals of the fluorescence and absorption spectra of the solvates with higher and lower frequencies of the electronic transition the energy migration appears to be directed from "blue" centers to "red" ones. This fact gives a clue to many spectroscopic features such as the long-wavelength shift of luminescence spectra at high concentration [4], the change of emission decay duration over the spectrum [4], the kinetics of luminescence spectra found in [6] and so on.

It is important to emphasize that according to eq. (1) an increase in the degree of environmental order corresponds to the enhancement of the electric field strength $\delta$ (at chaotic orientation of the molecules of the environment $\mathcal{E}=0$ ), i.e. to a decrease in the frequency of pure electronic transition of a molecule. Thus, the energy migration is directed from the centers with chaotic orientation of the environment to the centers with high order orientation of the media. It is assumed that such phenomena exist in biological structures; for instance, in plant photosynthetic structures between the pigment molecules. This problem is of special interest because the mechanism of directed transport of the excitation energy from antenna chlorophyll molecules to the reaction centers of photosynthetic apparatus in vivo is not yet clear.

In the present paper we are mainly concerned with investigations on the possibility of the directed non-radiative transfer of electronic energy (DNTEE) between the pigment molecules in a polymer matrix, i.e. in vitro. Two wellknown pigments, chlorophyll " $a$ " and pheophytin " $a$ " were chosen as samples. Both species are of great importance in the photosynthesis of the native systems, both in migration and in the primary processes of solar energy conversion into chemical energy of separated charges. 


\section{Experimental}

The pigment solutions were prepared as thin films at different concentrations but with the same optical density 0.2 at maximum absorption. The pigment molecules were dissolved in polyvinylbuthyral (PVB) $(n=1.489)$. The extraction and chromatographic purification of the pigments were performed using well-known standard techniques. The film fabrication was carried out by mixing the PVB alcohol solutions with the investigated pigment; alcohol was then evaporated in the dark for $24 \mathrm{~h}$ at temperature $T=20^{\circ} \mathrm{C}$. Thus, one can obtain films of different thicknesses (from 10-15 $\mu \mathrm{m}$ up to 50-60 $\mu \mathrm{m}$ and higher with increasing pigment concentration (from $5 \times 10^{-5} \mathrm{M}$ up to $4 \times$ $10^{-2} \mathrm{M}$ ) by varying the amounts of PVB, pigment and alcohol.

The measurements of the absorption spectra in the visible range were made using a standard spectrophotometer SF-10. The steady-state fluorescence spectra at different concentrations were recorded using a spectrofluorimeter "Fica55 " with automatic correction of spectral response. For the investigation of the instantaneous spectra and the decay kinetics of emission, the nanosecond laser spectrofluorimeter with $\mathrm{N}_{2}$ laser $\left(\lambda=337.1 \mathrm{~nm}, P_{\mathrm{m}}=500 \mathrm{kWt}, \tau_{\mathrm{FWHM}}=2.4 \mathrm{~ns}\right.$, repetition rate up to $150 \mathrm{~Hz}$ ) was used as the excitation source. The fast photomultiplier 18 ELCH-FM (0.7 ns time resolution), the C7-II sampling scope ( $6 \mathrm{GHz}$ frequency band), the $\mathrm{K}-20 \mathrm{I}$ recorder, and the $\mathrm{C} 8-2$ oscilloscope were employed for the monitoring of luminescence pulses. The lifetimes of the excited states were determined with the help of an analog computer, by comparing the calculated decay curves with the luminescence pulses observed experimentally [7]. The generation of the calculated decay curves coinciding with the fluorescent pulses under registration was done taking into account the real shape of the excitation pulse.

\section{Experimental results}

The experiments show that the steady-state and the temporal characteristics of luminescence of the pigments in rigid solutions are essentially dependent on the concentration. Fig. 1 presents the steady-state fluorescence spectra of chlorophyll "a" and pheophytin "a" in PVB at low (curves 1, 1") and high (curves 2,2') concentrations of pigment molecules when excited in the Stokes range. It is seen that the spectrum of the pigments at high concentrations is shifted towards the long-wavelength side (by $4.5 \mathrm{~nm}$ for chlorophyll and by 1.5 $\mathrm{nm}$ for pheophytin) with respect to the spectrum at low concentrations. The spectral line profile in fact remains the same in the region of the main fluorescence band. The investigations show that there exists a temporal shift of the emission spectra during $15 \mathrm{~ns}$ after switching on the excitation at high pigment concentrations. The "instant" luminescence spectra as well as the 

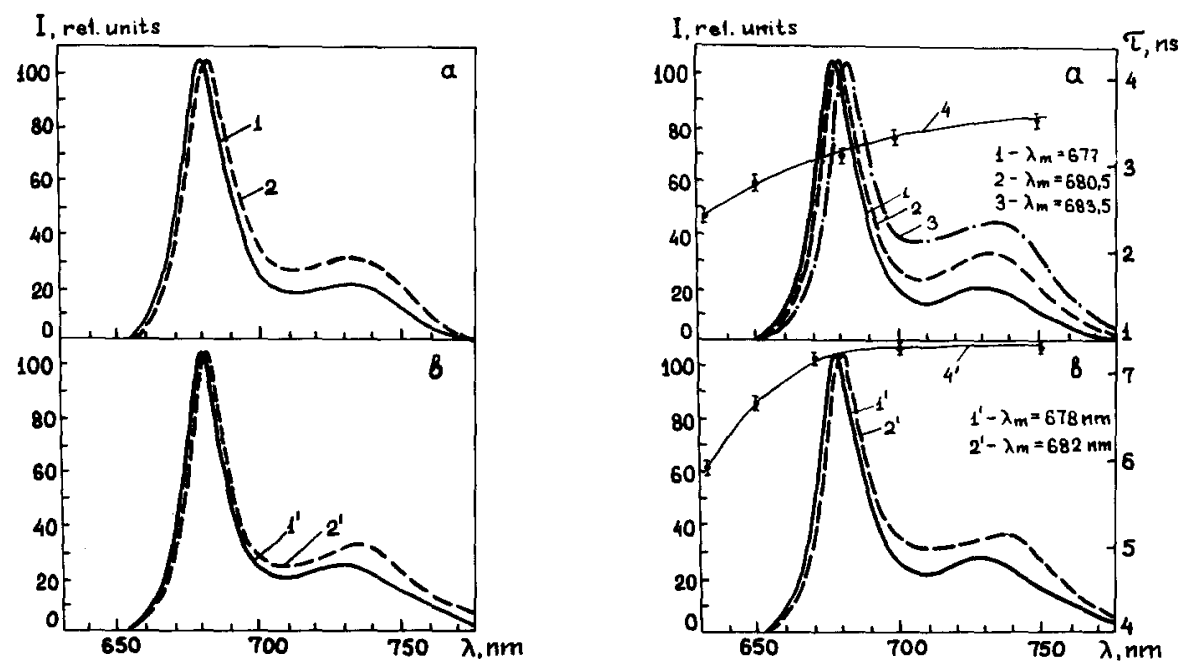

Fig. I. Integral luminescence spectra of the chlorophyll " $a$ " (a) and pheophytin " $a$ " (b) solutions in PVB at the Stokes excitation $\left(\lambda_{e x}=337 \mathrm{~nm}\right),(1) \quad c=10^{-3} \mathrm{M}\left(\lambda_{\mathrm{m}}=677 \mathrm{~nm}\right) ;(2) 4 \times 10^{-2} \mathrm{M}$ $\left(\lambda_{\mathrm{m}}=681.5 \mathrm{~nm}\right) ;\left(1^{\prime}\right) c=10^{-3} \mathrm{M}\left(\lambda_{\mathrm{m}}=678 \mathrm{~nm}\right) ;\left(2^{\prime}\right) c=4 \times 10^{-2} \mathrm{M}\left(\lambda_{\mathrm{m}}=679.5 \mathrm{~nm}\right)$.

Fig. 2. Instant luminescence spectra $\left(1,2,3\right.$ and $\left.l^{\prime}, 2^{\prime}\right)$ and luminescence duration spectra (4 and $\left.4^{\prime}\right)$ of chlorophyll " $a$ " (a) and pheophytin " $a$ " (b) solution in PVB at the Stokes excitation $\left(\lambda_{e x}=337\right.$ nm) (1) $c=10^{-3} \mathrm{M}, t=10 \mathrm{~ns}$; (2) $4 \times 10^{-2} \mathrm{M}, 2.5 \mathrm{~ns}$; (3) $4 \times 10^{-2} \mathrm{M}, 15 \mathrm{~ns}$; (1') $10^{-3} \mathrm{M}, 2.5 \mathrm{~ns}$; (2') $4 \times 10^{-2}, 15 \mathrm{~ns}$.

luminescence duration spectra for the pigments at high concentrations are presented in fig. 2. As is seen from fig. 2, the emission spectrum exhibits a monotonic temporal shift towards the long wavelength $(3 \mathrm{~nm}$ for chlorophyll during the period from 2.5 to $15 \mathrm{~ns}$, curves 2,3 ). It is interesting to note that the fluorescence spectrum of chlorophyll at high concentration (curve 2, fig. 2a) is shifted monotonically to the red part by $3.5 \mathrm{~nm}$ during $2.5 \mathrm{~ns}$ as compared with the instantaneous spectrum (curve 1, fig. 2a) of the solution at low concentration in which the spectral kinetics of the emission is absent. From fig. 2 it is also seen that the emission duration of the investigated samples grows monotonically as the monitored wavelength increases. In contrast with this the excited state lifetime in the case of low pigment concentrations is constant over emission spectrum and corresponds to $7.4 \mathrm{~ns}$ for chlorophyll and $7.6 \mathrm{~ns}$ for pheophytin.

\section{Discussion}

The summation of the results obtained proves the existence of the DNTEE owing to the inhomogeneous orientational broadening of the pigment energy levels in a polymeric matrix. 
First of all, the investigations in [8] definitely show that the energy migration between monomeric molecules of chlorophyll and pheophytin is caused by the dipole-dipole inductive resonance interactions. The data of table I support this conclusion. Table 1 shows the experimental and theoretical values of the critical radius $R_{0}$ of the energy transfer and presents the main physical constants of the system under investigation as well. As is seen from table 1 the agreement between theory and experiment is rather good in the range of the critical concentrations for chlorophyll molecules. Moreover, there is a good agreement between the theoretical and experimental results on the luminescence depolarization dependence on concentration of the chlorophyll molecules in PVB over a wide range of concentrations [9].

On the other hand, the data of the temperature dependences of the electronic spectra of chlorophyll and pheophytin in diluted solutions show a considerable orientational broadening of the energy levels for soluted pigments. Fig. 3 presents the integral luminescence spectra of pheophytin $(c=1 \times$ $10^{-5} \mathrm{M}$ ) in castor oil (polar solvent) having the same refractive index as PVB (taken from [9]). As is seen from fig. 3, cooling the pigment solution down to $-196^{\circ} \mathrm{C}$ leads to the hypsochromic shift of the spectrum by $3 \mathrm{~nm}$, with no change of the profile. Similar effects are observed in chlorophyll solutions testifying unambiguously the existence of orientational broadening of the energy levels in both pigment solutions.

As was previously mentioned, energy migration in the rigid solutions with orientational broadening of the electronic spectra is directed and goes from the solvates with higher pure electronic frequencies to the solvates with lower ones. The DNTEE causes the temporal shift of the spontaneous emission spectrum to the long-wavelength part as well as the concentration red shift of the luminescence integral spectrum and an increase of emission duration with the wavelength. As is shown the above effects are observed experimentally (fig. 1, curve 2).

Table 1

Spectral characteristics and transfer parameters for pigments in isotropic samples of PVB (refractive index $n=1.489$, orientational factor, $K^{2}=0.472$ )

\begin{tabular}{|c|c|c|c|c|c|c|}
\hline $\begin{array}{l}\lambda_{\max }^{\mathrm{abs}} \\
(\mathrm{nm})\end{array}$ & $\begin{array}{l}\lambda_{\max }^{\mathrm{abs}} \\
(\mathrm{nm})\end{array}$ & $\begin{array}{l}\text { Extinction } \\
\text { coefficient } \\
\mathcal{E}\left(\lambda_{\max }^{\text {abs }}\right) \\
\left(\mathbf{M}^{-1} \mathrm{~cm}^{-1}\right)\end{array}$ & $\begin{array}{l}\text { Quantum } \\
\text { yield of } \\
\text { luminescence } \\
B_{0}\end{array}$ & $\begin{array}{l}\text { Overlapping } \\
\text { integral } \\
\left(\mathrm{cm}^{6} / \mathrm{M}\right)\end{array}$ & $\begin{array}{l}R_{0}^{\text {exp }} \\
(\dot{\mathrm{A}})\end{array}$ & $\begin{array}{l}R_{0}^{\text {theor }} \\
(\dot{A})\end{array}$ \\
\hline \multicolumn{7}{|c|}{ Chlorophyll "a" } \\
\hline$\overline{677}$ & 669 & 66800 & 0.30 & $4.9 \times 10^{-13}$ & 48 & 48.2 \\
\hline \multicolumn{7}{|c|}{ Pheophytin "a" } \\
\hline 678 & 670 & 43100 & 0.21 & $2.8 \times 10^{-13}$ & - & 41.3 \\
\hline
\end{tabular}


When discussing the energy migration in the pigment solutions at high concentrations it is necessary to pay attention to the aggregation ability of chlorophyll " $a$ " and pheophytin " $a$ " molecules. In our investigations no traces

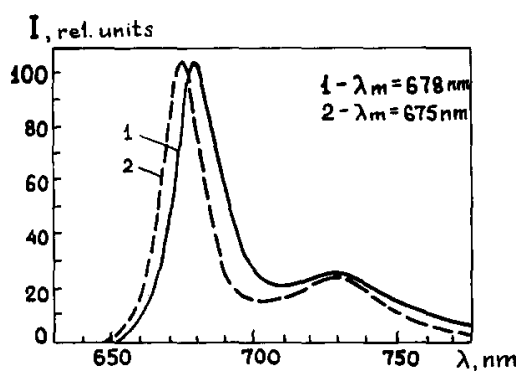

Fig. 3. Luminescence spectra of pheophytin " $\mathrm{a}$ " in castor oil $\left(A=0.2 ; \lambda_{\mathrm{ex}}=405 \mathrm{~nm}\right)(1) c=10^{-5} \mathrm{M}$, $T=20^{\circ} \mathrm{C}$; ) $c=10^{-5} \mathrm{M}, T=-196^{\circ} \mathrm{C}$

of aggregation in the pigment solutions in PVB with concentration up to $10^{-2} \mathrm{M}$ were observed. Increasing the pheophytin concentration up to $4 \times$ $10^{-2} \mathrm{M}$ leads to the appearance of a new absorption band in the range 693-700 $\mathrm{nm}$ and to the growth of the long-wavelength luminescence band intensity (fig. $1 b$ ). According to $[8,10,11]$ the pheophytin absorption in the range $693-700 \mathrm{~nm}$ at $c \sim 10^{-2} \mathrm{M}$ is ascribed to the pigment aggregates with weak luminescence in the spectral ranges $705-711 \mathrm{~nm}$ and $750-760 \mathrm{~nm}$. No changes in the absorption spectra of chlorophyll in PVB up to $c=4 \times 10^{-2} \mathrm{~m}$ were observed; i.e. the maximum of the main absorption band $(\lambda=669 \mathrm{~nm})$ is not shifted and the half-width of this band is constant $(\Delta \lambda=20 \mathrm{~nm})$. However, at $c=4 \times 10^{-2} \mathrm{M}$ the intensity of the long-wavelength luminescence band grows (fig. 1a) which gives evidence according to $[8,9]$ for the existence of the aggregates in solution with a luminescence band at $725-730 \mathrm{~nm}$. According to the investigations of the aggregation mechanisms of chlorophyll and its derivatives carried in [14,15], the formation of more short-wavelength aggregates of the pigment molecules with absorption at $673-678 \mathrm{~nm}$ is only possible in dry non-polar solvents. Thus, the pigment solutions in the present study at $c=4 \times 10^{-2} \mathrm{M}$ have molecular aggregates with the electronic spectra in the long-wavelength part with respect to the main luminescence band of their monomers. But, the intensity (quantum yield $\leqslant 1 \%$ ), shape and location $(\lambda>700 \mathrm{~nm})$ of the aggregate luminescence band of chlorophyll molecules [8-13] are such that they practically do not exert influence on the location and shape of the main luminescence band of monomers. The spectral distance between the main luminescence band of the monomers and the aggregate emission band is large enough and the absorption spectrum of the aggregates is situated between them. The absorption of luminescence light by the aggregates 
is exhibited as an "internal filter effect" and cannot explain the experimentally observed emission spectra kinetics.

Resuming the above one can draw a conclusion on the existence of the DNTEE between the monomeric molecules in the rigid polar solutions of the pigments which is caused by the dipole-dipole inductive resonant interactions between pigment molecules with orientational broadening of their electronic spectra and stimulates a number of spectroscopic effects. The presence of chlorophyll and pheophytin aggregates in concentrated solutions creates an additional way of the DNTEE from the monomers to their weak luminescence aggregates. The growth of the luminescence intensity at high concentrations in the spectral range $\lambda>700 \mathrm{~nm}$ for the steady-state spectra (fig. 1, curves $2,2^{\prime}$ ) indicates such DNTEE indirectly but the deformation of the "instant" luminescence spectra in the same range (fig. 2 , curves $3,2^{\prime}$ ) supports the above conclusion directly without any assumptions. It seems natural to connect this route of energy transfer and the concentration quenching of luminescence (CQL) in the systems under question. However, this process does not explain all the features of the quenching phenomenon. For instance, in the chlorophyll solutions CQL is stronger than in the pheophytin solution and the quantum yield drops down to $90.5 \%$ for chlorophyll and to $44 \%$ for pheophytin at the pigment concentrations $c=4 \times 10^{-2} \mathrm{M}$, though the quantum yields of monomer luminescence are of the same order for both the pigments. At the same time, as follows from the present paper and from [8,9], chlorophyll is not affected by aggregation as strongly as pheophytin. If one takes into account that the absorption of aggregates overlaps with the luminescence of a monomer which is stronger for pheophytin than for chlorophyll one can conclude that an additional route of non-radiative deactivation of an electronic state is needed for the explanation of the CQL. The DNTEE over the monomeric molecules from the blue solvates may be such a route. As a result of this DNTEE the excitation energy is trapped by the "red" centers which are more capable of non-radiative deactivating of the electronic states in comparison with the "blue" centers [16]. As follows from our investigations, the DNTEE over the monomeric molecules is greater for chlorophyll, i.e. one has the stronger CQL along the second route. This fact can explain the higher CQL of chlorophyll in comparison with pheophytin. The above-mentioned proposal about the nature of CQL for pigments demands further experimental examination.

It has been well-established that chlorophyll and its bacterial analogs can be observed in a cell in the form of several species differing by their behaviour due to the pigment-pigment interactions and interactions of pigment molecules with an environment (the monomeric species are in form of the short wavelengths and the aggregated species are in form of the long wavelengths). Besides, it has been shown that the photosynthesis is accomplished by at least two different photosystems incorporating spectrally different chlorophyll species and accompanying pigments. Instead of the single short-wavelength 
band of chlorphyll "a" typical for the solution, a number of bands related to various native species of chlorophyll "a" appear. The shortest wavelength band is close to the solution one while the others are situated in a longer wavelength range $(660-740 \mathrm{~nm})$ [17]. A similar situation takes place in the luminescence spectra as well.

The discovery of the photosynthetic unit poses the problem of how the energy of electronic excited states it transferred to the reaction centers (RC). The direct light absorption by the RC is negligible because of its small concentration. The majority of the molecules in the photosynthetic unit works like an antenna. The molecules of the antenna absorb the light quanta, the absorbed energy migrates along the antenna matrix and is finally transferred to the pigment complex in the $\mathrm{RC}$, where the photochemical reaction takes place.

The energy absorbed in the antenna matrix by the blue species migrates to the red centers which emit fluorescence light. This directivity of the migration (the energy transfer to the red centers) is explained by the mutual locations of electronic levels of various aggregated forms. The analysis of the emission and absorption maximal locations shows that the level sequence in different aggregates of chlorophyll "a" in vivo forms a descending ladder from the blue to the red forms [17]. The effective migration may be accomplished only in a downward direction because the back run process proceeds with overcoming an energy barrier. Such a property provides a very important function, i.e. the spacial concentration of absorbed energy in the locations connected with the $\mathrm{RC}$ of the photosynthesis. One can assume that the inhomogeneous broadening of electronic energy levels owing to the orientational interactions promotes the DNTEE in downward direction inside each of the aggregated forms of the pigment and especially in the short wavelength species (see fig. 4). Thus, the chaotic diffusion of excitation in the system of molecules with identical energy levels (homogeneous migration) is replaced in the case of inhomogeneous broadening by a directed movement towards the red centers which shortens the excitation migration way and is likely to decrease the deactivation probability in the antenna system of chlorophyll molecules. Hence, the existence of

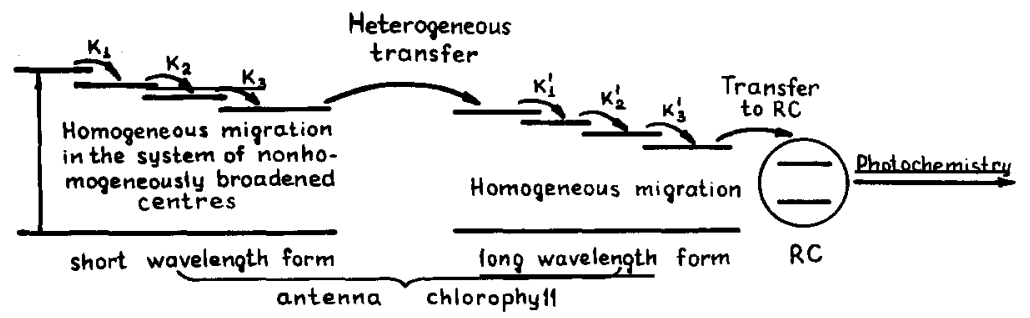

Fig. 4. Scheme of the electronic excitation energy transfer in an antenna complex with provision for both homogeneous and inhomogeneous migration. 
chlorophyll native forms in vivo together with the inhomogeneous broadening of electronic levels inside individual forms cause the directed relaxation of the antenna excitation in a downward direction in the vicinity of the RC.

\section{Conclusion}

(1) The results obtained testify the existence of DNTEE over monomeric molecules in rigid solutions of photosynthetic pigments at high concentrations due to orientational broadening of their electronic spectra. The DNTEE leads to a number of spectroscopic effects such as a long wavelength shift of integral luminescence spectrum, the kinetics of spontaneous emission spectrum in the nanosecond time scale and the monotonic growth of the emission duration with the wavelength. All these effects have been observed at high concentrations of the pigment molecules.

(2) A non-radiative energy transfer from the monomeric molecules to their aggregates is demonstrated by the temporal deformation of the luminescence spectra of pigment solutions at high concentrations.

(3) The DNTEE in both above-mentioned cases leads to the concentrational quenching of the pigment luminescence.

(4) Though the DNTEE was observed for the photosynthetic pigments in vitro, a similar process is expected for the pigments in vivo as well. In particular, the long-wavelength shift of the ethiolated sprouts [8] while greening may be explained by this process.

(5) The DNTEE between the molecules with orientationally broadened spectra is of special interest as it may provide the energy transport from low to high organized (in the sense of the environment orientation) structures in biological systems.

\section{References}

[1] N.G. Bahshiev, Spektroskopiya Mezhmolekulyarnyh Vzaimodeystivii (Nauka, Leningrad, 1973) p. 262.

[2] V.I. Tomin, Opt. i Spektr. 38 (1975) 274.

[3] A.N. Rubinov and V.I. Tomin, Opt. i Spektr. 29 (1970) 1082.

[4] I.M. Gulis and A.I. Komyak, Zh. prikl, spektr. 27 (1977) 841.

[5] I.M. Gulis, A.I. Komyak and V.I. Tomin, Izv. AN SSSR, Ser. Phys. 42 (1978) 307.

[6] N.A. Nemkovich, A.N. Rubinov and V.I. Tomin, 3rd Conf. on Luminescence, Conf. Digest., Szeged, Hungary, 1 (1979) p. 99.

[7] N.A. Nemkovich, V.M. Matzeiko and V.I. Tomin, Kvant. elektr. 7 (1980) 566.

[8] E.I. Zen'kevich, A.P. Losev and G.P. Gurinovich, Migratiziya energii Mezhdu pigmentami photosynteticheskogo apparata rastenii Preprint Insituta physiki AN BSSR, Minsk (1971) p. 60 .

[9] E.I. Zen'kevich, Migratziya energii elektronnogo vozbuzhdeniya $v$ Rastvorah photosyteticheskih pigmentov. Cand. diss., Minsk (1973) p. 191. 
[10] G.P. Gurinovich, A.N. Sevchenko and K.N. Solov'ev, Spektroskopiya Chlorophylla i rodstvennyh soedinenii, Minsk, Nauka i tekhnika (1968) p. 439.

[11] E.I. Zen'kevich and M.V. Sarzhevskaya, Biophysika (Dep. V VINITI) 24 (4) (1979) 771.

[12] C. Balny, S.S. Brody and G. Hui Bon Hoa, Photochem. Photobiol. 9 (1969) p. 445.

[13] S.B. Broyde, S.S. Brody and M. Brody, Biochem. Biophys. Acta 153 (1968) 183.

[14] L.L. Shipman, T.M. Cotton, J.R. Norris and J.J. Katz, J. Am. Chem. Soc. 98 (1976) 8222.

[15] G.P. Gurinovich, A.P. Losev and E.I. Sagun, Zh. Prikl. Spektr. 26 (1977) 1028.

[16] M. Ya. Kostko, L.G. Pikulik and V.A. Yakovenko, Zh. Prikl. Spektr. 15 (1971) 864.

[17] F.F. Litvin, v sbornike Biophysika Photosinteza (Moskva, Izd-vo MGU, glava I) p. 6-123. 